\title{
CONTOUR GENERATION BY WAVEFRONT RECONSTRUCTION
}

\author{
K. HAINES and B.P. HILDEBRAND \\ Institute of Science and Technology, The university of Michigan, \\ Ann Arbor, Michigan 48107, USA
}

Received 5 August 1965

The wavefront reconstruction technique, first proposed by Gabor [1] and later refined by E. N. Leith and J. Upatnieks [2] provides a method for storing three-dimensional information on a twodimensional storage surface. The storage is accomplished by recording the interference pattern of a reference wave of coherent monochromatic light with the scattered light from the three-dimensional object. Thus, the photographic plate contains a grating-like structure embodying both phase and amplitude information about the object. The information is retrieved by illuminating the developed plate with coherent monochromatic light. The structure on the plate acts like a diffraction grating. The first order diffraction terms reconstruct the three-dimensional object with great fidelity. One of these terms produces a true image while the other reconstructs a pseudoscopic image. The true image reveals all the three-dimensional properties normally attributed to the real world. In addition, magnification of the image may be achieved [3].

A possible application of this process is in the three-dimensional mapping of terrain or of models of certain manufactured goods. In such an application the addition of constant depth contour lines on the image would be extremely useful. This may be done by making an analogy with radar theory.

A hologram may be likened to a radar system where the photographic plate corresponds to a two-dimensional phased array antenna. Such an antenna has a depth of focus associated with it just as has the hologram. This depth of focus is an inverse function of the aperture size. In radar practice, however, a much greater depth or range resolution is achieved by broadbanding the illuminating signal. This technique from radar theory may be borrowed to provide a finer depth measurement than is obtainable from the aperture alone. In fact, if the right kind of broadbanding is used, contours of constant depth may be obtained on the image. To achieve this effect the illuminating source must consist of two distinct frequencies.

Radar theory predicts that the signal return will have a specific shape known as the ambiguity function of the transmitted signal [4]. If the signal is the sum of two continuous waves at different frequencies, the ambiguity function is a periodic function at the difference frequency. In terms of the wavef ront reconstruction, there will be fringes on the image. A closed fringe indicates a contour of constant range from the viewing point to the object. The range distance between fringes is a well-known expression used in radar, $\Delta R=\Delta f / f^{2}=\lambda^{2} / \Delta \lambda$, where $\Delta f=$ frequency difference, and $f=$ average frequency. As an example, suppose the depth interval between fringes is required to be one $\mathrm{mm}$. Then, if the wavelength of the light is $6328 \AA$, the wavelength separation must be approximately $4 \AA$. For coarser fringe spacing the wavelength separation becomes smaller. Laser technology has advanced to the state where several frequencies are now available. Thus this method becomes quite practical.

A simple optical explanation of the effect described above is as follows. The two frequency illumination produces two interference patterns on the photographic plate. That is, two holograms are made simultaneously. The developed plate is then illuminated with light at a single frequency. Thus two images are formed due to the two holograms. However, one image is magnified by an amount different than the other, since magnification depends upon the ratio of the frequency of the reconstruction source to the frequency of recording the source [3]. Since depth magnification is the square of the lateral magnification, the distance between identical points in the two images becomes a function of depth. Light from the two points interferes either destructively or constructively depending upon the distance between them. Thus at a par- 
ticular depth or range, a bright contour becomes visible. The next fringe indicates a contour at a different range. If the equations are worked out on this optical basis, the expression for range separation between fringes is identical to that quoted from radar theory.
2. E.N. Leith and J. Upatnieks, J.Opt. Soc. Am. 52 (1962) 1123; 53 (1963) 1377; 54 (1964) 1295.

3. E.N. Leith, J. Upatnieks and K. Haines, Microscopy by wavefront reconstruction, to be published in J.Opt.Soc.Am. (1965).

4. P.M. Woodward, Probability and information theory with applications to radar (Pergamon Press, Ltd., London).

1. D.Gabor, Nature 161 (1948) 777; Proc. Roy. Soc. (London) A197 (1949) 454. \\ ELECTRON TEMPERATURE MEASUREMENTS IN A MEGAJOULE \\ THETA-PINCH BY LIGHT SCATTERING * \\ H. -J. KUNZE, E. FÜNFER and H. RÖHR \\ Institut fur Plasmaphysik, Garching b.München, Germany
}

Received 5 August 1965

In the present communication we wish to report the successful application of light scattering to the measurement of the electron temperature in a large theta-pinch.

During the last two years this new diagnostic method has proved to be very powerful, and contrary to other methods it yields local plasma parameters without disturbing the plasma. After developing this method in a small pinch [1] and applying it to a somewhat larger one [2], it is now used for the investigation of the megajoule theta-pinch (Isar I) at Garching.

The data of this pinch shall be given elsewhere. For the observation of the scattered radiation the coil has in the middle a conical hole with a diameter of $6 \mathrm{~mm}$ at the inner surface of the coil. The light source is a ruby laser of the Raytheon Company. It is Q-switched by means of a Pockels cell and is operated at an output between 100 and $200 \mathrm{MW}$ with a pulse length of about $40 \mathrm{nsec}$. By means of a telescopic system the laser beam is focused along the axis of the discharge tube, and has a spot diameter of $5 \mathrm{~mm}$ at the observed plasma volume. With this arrangement the scattered radiation is observed at right angles to the incident beam.

The formerly used measuring arrangement [1] was developed further and considerably im-

\footnotetext{
* This work was performed as part of the joint research program of the Institut fitr Plasmaphysik, Garching and Euratom.
}

proved. The main part is a monochromator in Littrow mounting, equipped with a multiple exit slit of glass fibers developed by E. Glock [3]. One of these fiber slits is used as entrance slit, whereas the other ones serve in the usual manner as exit slits. Besides the fact that this arrangement is very simple and that the entrance slit image at the exit slits is of high optical quality, the main advantage of this device is that the other end of the fiber bundle which serves as entrance slit may have any desired cross section. Now the observed volume in the plasma can

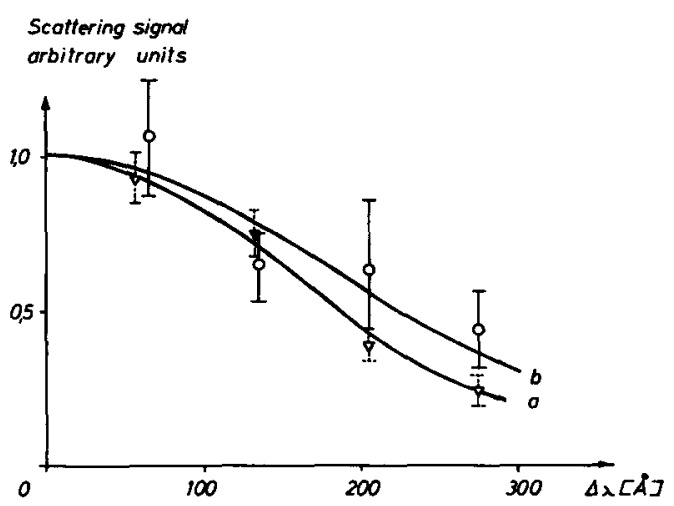

Fig. 1. Curve a: Spectrum of scattered light $1.3 \mu \mathrm{s}$ after ignition. Bank energy 0.33 MJoule, initial pressure $10 \mathrm{uD}_{2}$, electron temperature $134+17 \mathrm{eV}$. Curve b: Spectrum of scattered light $1.1 \mu \mathrm{s}$ after ignition. Bank energy 1.5 MJoule, initial pressure $10 \mathrm{uD}_{2}$, electron temperature $215 \pm 115 \mathrm{eV}$. 